\title{
Genogram: tool for exploring and improving biomedical and psychological research
}

\author{
Genograma: una herramienta para explorar y mejorar la investigación biomédica y psicológica \\ Jorge Mauricio Cuartas Arias ${ }^{1 *}$
}

${ }^{1}$ Faculty of psychology, Universidad de San Buenaventura, Medellín, Colombia.

*Corresponding author: mauricio.cuartas@usbmed.edu.co

Currently, genograms have become a basic and fundamental tool used to graphically represent a detailed record of family information. Their construction should include not only basic details and family structure, but also information that can outline different approaches for both research and clinical intervention.

In basic and biomedical research, genealogies are a fundamental resource for the evaluation of genetic homogeneity of a population or sample group. Their use can help researchers understand maternal and paternal lineages, the diversity of last names, and the probable level of genetic mixing within a family or group of families settled in a determined place. In this regard, advances in our understanding of the genetics of human illnesses, rooted in population genetics, has encouraged comparative studies of genetic variation between individuals of the same population using a mathematical approximation of the allele distribution or genetic variants of interest to the researcher. This approach also motivates the detailed construction of family trees and pedigree charts. Detailed pedigree analysis can help in the detection of the dominance and recessiveness of a given trait, which comes from the study of phenotypes and helps identify Mendelian diseases with dominant or recessive patterns. For example, in cognitive performance congenital errors of metabolism are frequently associated with the presence of mental retardation; these have also been associated with recessive inheritance patterns. On the other hand, the high inheritability of mental disorders expressed in studies of family aggregation and identical twins reflects the importance of meticulously constructing family trees to establish the following: consanguinity and its probable contribution to the likelihood of the presence of various syndromes associated to cognitive performance; the founding ancestors in a family tree, particularly the diversity of last names and how this suggests a particular genetic structure; and levels of genetic mixing, allowing for an estimation of the proportions of other populations and their differential contribution to the development of diseases within a particular environmental context.

Additionally, genograms allow us to make inferences about prognoses, the presence of distinctive clinical subtypes, and the evolution of symptoms. This is possible when clinical patterns or frequent behaviors can be identified in a patient and are shared with other close family members. One example of this is the findings on neurocognitive performance in the index case and other family members, suggesting the presence of candidate endophenotypes in different domains of executive function in mental illnesses like schizophrenia, attention deficit hyperactivity disorder, type one bipolar disorder, autism, and Gilles de la Tourette syndrome, among others. Genograms have also allowed for advances in the study of CADASIL, Alzheimer's disease, Parkinson's, and other neurodegenerative diseases which show family transmission. Thanks to the detailed process of pedigree construction, researchers have been able to identify genetic modifications, modifier genes, and variable cognitive performance as a fundamental resource to aid our understanding of the physiopathology of these diseases. This approach has also generated important information allowing for advances in preliminary diagnoses, clinical evolution, and differential pharmacological responses.

Even beyond their use in basic research and the estimation of inheritability, including for complex traits like psychiatric illnesses and psychopathology, genograms are a key tool in family therapy. The use of genealogy contributes to the recognition of critical family events, codifying and symbolizing the recording of family relations. They orient the therapist toward incidents with greater emotional charge, helping him detect the traumatic experiences of previous generations that are known as transgenerational trauma and can be major determining factors in personality construction. This allows for two crucial findings: the recognition of cognitive robustness that family members use to overcome stress and develop resiliency in specific contexts or situations of change; and the identification of key family members to support dynamics of 
change to encourage healthy lifestyles or discourage behavior that is disruptive to the family like drug or alcohol consumption, impulse control, and other conduct that affects family ties, performance, and individual well-being.

Although the use of genograms has been fundamental within systems theory to study the impact of interrelations, attachment links, mental health, and the shaping potential of the underlying family construct, in practice clinical psychologists often neglect to condense this information through the use of genograms. This delays psychotherapeutic intervention because the patient's narrative will not always lead to the detection of the nuclei of family conflict and their evolution with an eye towards understanding the development of discomfort in the patient (or the index case in the genogram). As a result, the lack of detailed comprehension of the group and family dynamic (for which there are an abundance of graphic representations available online) affects the formulation of hypotheses within an adequate time frame and complicates an appropriate psychodiagnosis to deal with the patient's personality and the direct and indirect influences on his psychological and social functioning.

For all these reasons, it's essential that the processes of psychological and psychiatric attention begin with the construction of the patient's genogram. This will form the basis for the development of biomedical research and the treatment of complex social and psychological phenomena in a more efficient way, reducing intervention times and improving therapeutic results. 